\title{
Impact of Joule heating on the stability phase diagrams of perpendicular magnetic tunnel junctions
}

\author{
N. Strelkov, ${ }^{1,2, *}$ A. Chavent,${ }^{1}$ A. Timopheev,${ }^{1}$ R. C. Sousa,${ }^{1}$ \\ I. L. Prejbeanu, ${ }^{1}$ L. D. Buda-Prejbeanu, ${ }^{1}$ and B. Dieny ${ }^{1, \dagger}$ \\ ${ }^{1}$ Univ.Grenoble Alpes, CNRS, CEA, Grenoble INP, INAC-SPINTEC, 38000 Grenoble, France \\ ${ }^{2}$ Department of Physics, Moscow Lomonosov State University, Moscow 119991, Russia
}

\begin{abstract}
Measured switching voltage-field (V-H) diagrams of perpendicular magnetic tunnel junctions (pMTJ) exhibit unexpected behavior at high voltages associated with significant heating of the storage layer. The boundaries deviate from the critical lines corresponding to the coercive field, which contrasts with the theoretically predicted behavior of a standard macrospin-based model. Combining recent experimental studies of the temperature dependence of spin polarization and perpendicular magnetic anisotropy (PMA) we are proposing a modified model. Our approach takes into account the Joule heating during the writing pulse, which reduces the spin polarization and the anisotropy, thereby reducing the spin torque efficiency and the coercive field during the switching. The numerical macrospin simulations based on this model are in agreement with our experimental measurements and consistent with the results derived from the linearization of Landau-LifshitzGilbert equation.
\end{abstract}

Keywords: Spintronics, MRAM, STT switching, phase diagrams, Joule heating, LLG linearization

\section{INTRODUCTION}

A large number of studies related to spin transfer torque (STT) switching $[1,2]$ in magnetic tunnel junctions (MTJ) were conducted over the past few years in support of the development of spin transfer torque magnetic random access memory (STT-MRAM). This memory uses as storage elements $\mathrm{MgO}$-based magnetic tunnel junctions with perpendicular (i.e. out-of-plane) magnetization (pMTJs) [3, 4]. The common stacks involve $\mathrm{MgO} / \mathrm{FeCoB}$ interfaces allowing to reach the technological requirements in terms of magnetoresistance variation around $200 \%[5,6]$ and thermal stability factor at room temperature around $60[7,8]$. The main properties of the memory MTJ cell can be extracted from their writing voltage-field ( $\mathrm{V}-\mathrm{H})$ diagram [9]. Experimental observations and theoretical modelling of such diagrams in collinear [6, 10-12] and non-collinear geometries [13] give an almost complete description of STT-induced switching processes in MTJ. However, at high voltages (typically above $0.4 \mathrm{~V}-0.8 \mathrm{~V}$ depending on the junction resistance), most experimental diagrams show deviation from the theoretically predicted behavior. A plausible origin of this effect is the heating due to Joule dissipation around the tunnel barrier and the resulting variation of the storage layer magnetic properties. It is well-known that the coercive field of magnetic layers decreases with temperature $[14,15]$. STT reduction due to the heating was also noticed earlier in experiments on current-induced domain wall motion $[16,17]$ and it was observed both in spin valves [18] and MTJs [19]. Bandiera et al. proposed a concept of thermally assisted STT-MRAM in which the heating due to the STT write current is also used to induce an anisotropy reorientation from out-ofplane to in-plane [20]. Moreover, recent experiments have shown that the $\mathrm{MgO} / \mathrm{CoFeB}$ interface with thin $\mathrm{MgO}$ tunnel barrier have a much lower (more than two orders of magnitude) thermal conductivity than what could be expected from the bulk value [21]. In double barrier MTJs in which the storage layer is sandwiched between two $\mathrm{MgO}$ barriers, this further enhances the Joule heating of the storage layer.

In this paper, we present a series of experimental studies and their theoretical interpretation using the macrospin model that takes into account the heating effects described above. These results point out the strong influence of the Joule heating on switching abilities of MTJs at high voltages. Considering the importance of these heating effects, it is mandatory to include these heating effects in MRAM design tools.

\section{EXPERIMENTS}

We performed a number of experiments using different pillars based on pMTJ stack with different compositions and sizes. Typical switching diagrams are summarized in FIG. 1 for various kind of samples varying the composition of the stack and the nanofabrication procedure (see TAB I). The wafers in FIG. 1(a,b,d) were grown by $\mathrm{dc}$ and $\mathrm{rf}$ magnetron sputtering on a thermally oxidized Si substrate. The sample from FIG. 1(c) was grown on pre-patterned conducting non-magnetic pillar without post-deposition etching [22].

The storage layer from FIG. 1(a,b,d) consists of two ferromagnetic parts separated by a thin $\mathrm{W}$ film and sandwiched between two $\mathrm{MgO}$ layers to increase the interfacial anisotropy: the main $\mathrm{MgO}$ barrier which provides the TMR and a thinner MgO layer with lower resistancearea (RA) product. Next to the $\mathrm{MgO}$ layer, there is a 

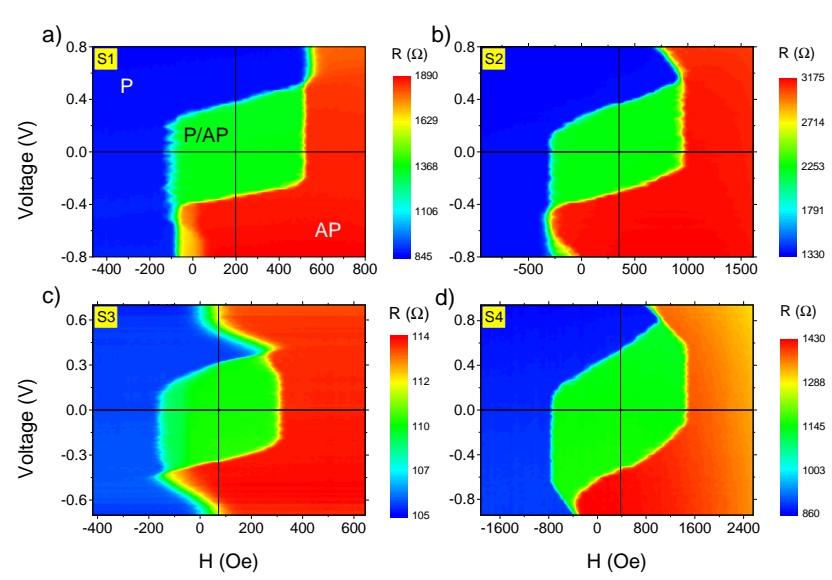

FIG. 1. Experimental stability V-H diagrams for the different samples based on pMTJ stacks. The color scale corresponds to the resistance of pMTJ. Field shift with respect to the $H=0$ Oe corresponds to an uncompensated stray field $H_{\text {stray }}$ from the SAF (a) 12 R-H loops, $H_{\text {stray }}=192$ Oe, $\mathrm{TMR}=124 \%$ (b) $12 \mathrm{R}-\mathrm{H}$ loops, $H_{\text {stray }}=352$ Oe, $\mathrm{TMR}=139 \%$ (c) $12 \mathrm{R}-\mathrm{H}$ loops, $H_{\text {stray }}=72 \mathrm{Oe}, \mathrm{TMR}=9 \%$ (d) $10 \mathrm{R}-\mathrm{H}$ loops, $H_{\text {stray }}=379$ Oe, $\mathrm{TMR}=66 \%$. See sample details in TAB. I.

TABLE I. Stack composition of the used samples

\begin{tabular}{llr}
\hline$\#$ & Figure & Sample description \\
\hline \hline S1 & FIG. 1(a) & SAF $/ \mathrm{MgO} / \mathrm{FeCoB}(1.3 \mathrm{~nm}) / \mathrm{W}(0.3 \mathrm{~nm}) /$ \\
& & $\mathrm{FeCoB}(0.5 \mathrm{~nm}) / \mathrm{MgO} / \mathrm{Capping}$, \\
& & $D=110 \mathrm{~nm}, \mathrm{RA}=10 \Omega \cdot \mu \mathrm{m}^{2}$ \\
\hline S2 & FIG. 1(b), $\mathrm{SAF} / \mathrm{MgO} / \mathrm{FeCoB}(1.3 \mathrm{~nm}) / \mathrm{W}(0.3 \mathrm{~nm}) /$ \\
S2b & FIG. 2, & $\mathrm{FeCoB}(0.5 \mathrm{~nm}) / \mathrm{MgO} / \mathrm{Capping}$, \\
& FIG. 7(b) & $D=80 \mathrm{~nm}, \mathrm{RA}=10 \Omega \cdot \mu \mathrm{m}^{2}$ \\
\hline S3 & FIG. 1(c), & $\mathrm{SAF} / \mathrm{MgO} / \mathrm{FeCoB}(1.5 \mathrm{~nm}) /$ \\
& FIG. 6 & $\mathrm{W}(2 \mathrm{~nm}) / \mathrm{Capping}$, \\
& FIG. 7(a) & $D=300 \mathrm{~nm}, \mathrm{RA}=9 \Omega \cdot \mu \mathrm{m}^{2}$ \\
\hline S4 & FIG. 1(d) & $\mathrm{SAF} / \mathrm{MgO} / \mathrm{FeCoB}(0.9 \mathrm{~nm}) / \mathrm{W}(0.1 \mathrm{~nm}) /$ \\
S4b & FIG. 3 & $\mathrm{Co}(0.5 \mathrm{~nm}) / \mathrm{W}(0.1 \mathrm{~nm}) / \mathrm{FeCoB}(0.8 \mathrm{~nm}) /$ \\
& & $\mathrm{Cappng}, D=70 \mathrm{~nm}, \mathrm{RA}=9 \Omega \cdot \mu \mathrm{m}^{2}$ \\
\hline
\end{tabular}

thin $\mathrm{W}$ capping layer is inserted to absorb the $\mathrm{B}$ away from the $\mathrm{MgO}$ interfaces upon annealing [6, 12, 23]. The magnetization of the bottom reference layer is pinned by a synthetic antiferromagnet (SAF).

The measurement process to derive the $\mathrm{V}-\mathrm{H}$ diagrams is the same as described in Ref. [11]. A magnetic field is applied out-of-plane, i.e. along the easy axis of magnetization of the storage layer and slowly varied (typically $50 \mathrm{kOe} / \mathrm{s}$ ) after each write-read sequence. A $100 \mathrm{~ns}$ writing voltage pulse of defined amplitude is applied at each magnetic field point. The resistance is measured under low constant (dc) current after applying the writing pulse. The resistance-field (R-H) loop is repeated several times, and the final resistance value is defined by averaging all these loops.

In the FIG. 1, one can see V-H diagrams with various shapes. Three main stability areas are identified: the high-resistance area with antiparallel (red, AP) configuration of storage and reference layer, the low-resistance area with parallel (blue, P) configuration and bistable (green, $\mathrm{P} / \mathrm{AP}$ ) area where both mentioned configurations are possible. The center of the bistable area is shifted with respect to the zero field. This shift corresponds to an uncompensated stray field $H_{\text {stray }}$ from the SAF.

The shape of the diagram in FIG. 1(a) is predictable by the theory developed in Ref. [11] - there are straight vertical boundaries corresponding to the coercive field. But the three other diagrams show deviations from the theoretically expected shape. This unpredictable behavior can be due to Joule heating effects inside the storage layer. A number of recent experiments supports this interpretation. For example, it was observed earlier that the STT write current flowing through pMTJs could induce a decrease of both PMA and coercivity due to Joule heating around the tunnel barrier [20]. In FIG. 1(c), it can be clearly seen that the critical lines lean back towards the $H_{\text {stray }}$ value at high voltages and completely coincide with $H_{\text {stray }}$ when the absolute value of the applied voltage exceeds $\sim 0.6 \mathrm{~V}$. This could mean that the PMA and spin polarization in storage layer are reduced by Joule heating to the point where the critical lines then correspond to the applied field value which compensates the SAF stray field $H_{\text {stray }}$. Further comparing FIG. 1(a) and FIG. 1(b), a stronger thermal influence can be noted in the smaller junction. This can be explained by considering that smaller samples should be more sensitive to the temperature variation, as the stability factor of MTJ in macrospin approximation $\Delta=K_{\mathrm{eff}} \cdot \Omega /\left(k_{B} T\right)$ depends on the volume $\Omega$ of the storage layer, $K_{\text {eff }}$ being the effective perpendicular anisotropy constant.

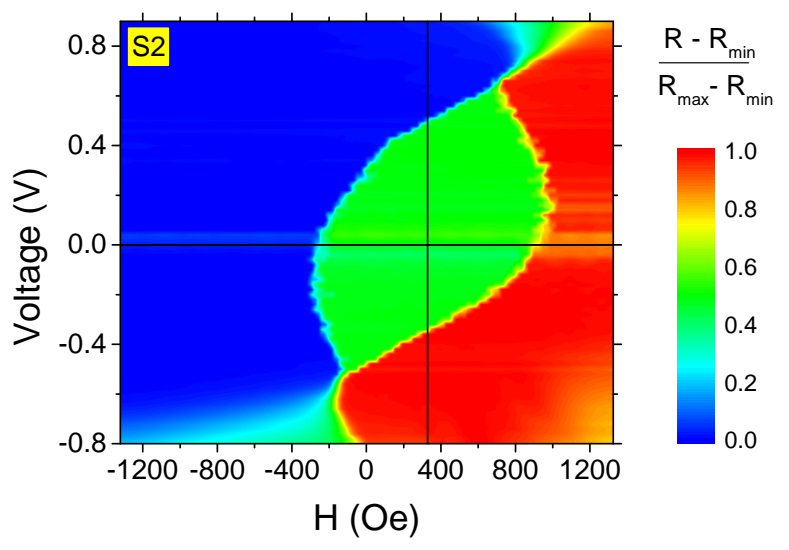

FIG. 2. Normalized experimental stability V-H diagram measured under constantly applied (dc) voltage. Sample is the same as in FIG. 1(b) (see details in TAB. I).

For a given voltage, one might expect that the longer 
the writing pulse, the higher the temperature reached in the storage layer and therefore stronger the impact of the heating. To check this effect on our samples, the measurement procedure was changed. A constant (dc) voltage was applied to the sample and the applied field varied step by step from -1400 Oe to 1400 Oe and vice versa. At each field step, the current was measured during $500 \mu \mathrm{s}$ and the resulting resistance calculated as $R=V_{\text {bias }} / I$. In FIG. 2, the normalized experimental V$\mathrm{H}$ diagram measured using this procedure is shown. As can be seen, there are no more vertical straight boundaries corresponding to field equal to coercive field. Since here the current is steadily flowing through the sample, the magnetic tunnel junction cannot cool down between each resistance measurement, so that a significant decrease of the anisotropy due to Joule heating takes place as the bias voltage is gradually increased.

To estimate the temperature variation and its voltage dependence in MTJs, we performed an experiment by the method described in Ref. [24] based on the temperature dependence of RKKY coupling, using the sample similar to that from FIG. 1(d) but with larger diameter $D=170 \mathrm{~nm}$. We measured the $\mathrm{V}-\mathrm{H}$ diagram under dc voltage and extracted the voltage dependence of spin-flop field $H_{\mathrm{SF}}$ (white solid line in FIG. 3(a)), at which both SAF layers become magnetized in parallel configuration versus applied voltage. This dependence is parabolic, and its expression can be derived from FIG. 3(b). We then measured the variation of $H_{\mathrm{SF}}$ field versus controlled temperature which is found to be almost linear FIG. 3(c). By combining the data from FIG. 3(b) and FIG. 3(c), the temperature elevation $\Delta T$ due to current flow versus applied voltage can be derived. A quadratic variation is obtained given by $\Delta T=308 \cdot V^{2}$ and shown in FIG. 3(d) ( $T$ in $\mathrm{K}, V$ in volt).

On the $\mathrm{V}-\mathrm{H}$ diagrams in FIG. 1 one can note some asymmetry between the critical lines for positive and negative voltage polarities. For example, the absolute value of switching voltage $V_{\mathrm{sw}}$ at $H=H_{\text {stray }}$ (black vertical line) corresponding to zero applied field, is different for positive $\left(V_{\mathrm{sw}}^{+}\right)$and negative $\left(V_{\mathrm{sw}}^{-}\right)$voltages. One of the possible reasons for such asymmetry might be due to the different resistances for P-AP and AP-P transitions. If the heating power at fixed voltage reads as $V^{2} / R$, then lower resistance causes more heating and, as a result, a greater decrease of PMA and spin polarization. In FIG. 1(a), the TMR amplitude is $124 \%, V_{\mathrm{sw}}^{+}=0.37 \mathrm{~V}$ (AP-P transition with high resistance and lower temperature), $V_{\mathrm{sw}}^{-}=0.33 \mathrm{~V}$ (P-AP transition with low resistance and higher temperature). The switching voltage difference for this sample is $12 \%$. In FIG.1(b), the TMR amplitude is $139 \%, V_{\mathrm{sw}}^{+}=0.37 \mathrm{~V}, V_{\mathrm{sw}}^{-}=0.3 \mathrm{~V}$ and difference is $23 \%$. For the sample with TMR amplitude $66 \%$ in FIG.1(d), $V_{\mathrm{sw}}^{+}=0.54 \mathrm{~V}, V_{\mathrm{sw}}^{-}=0.52 \mathrm{~V}$ and the difference is $4 \%$. So, we can state that the sample with larger TMR amplitude have more pronounced asymmetry in its V-
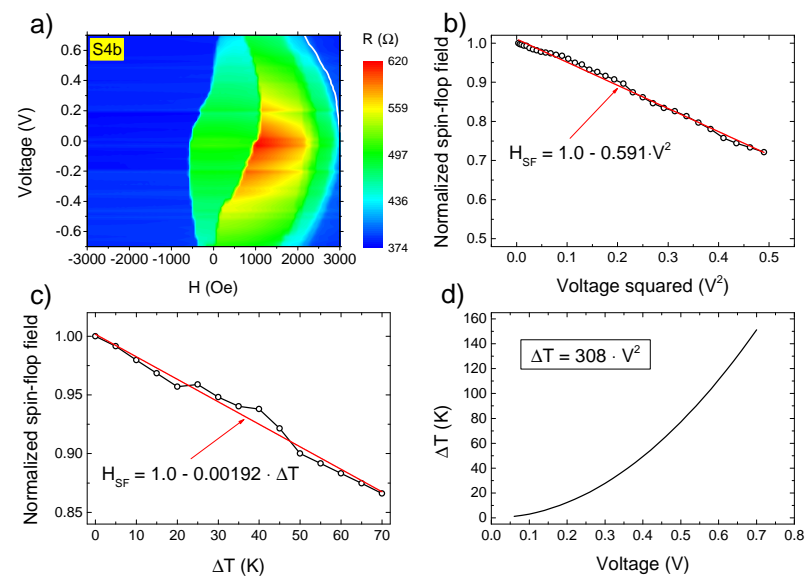

FIG. 3. (a) Experimental stability V-H diagram measured under dc voltage for the sample similar to that from FIG. 1(d) with $D=170 \mathrm{~nm}$ and extracted spin-flop field-voltage dependence (white solid line). (b) Linear fit of normalized spin-flop field-voltage squared dependence. (c) Linear fit of normalized spin-flop field-temperature variation dependence. (d) Temperature elevation due to current flow versus voltage derived by combining the fits from FIG. 3(b) and FIG. 3(c).

$\mathrm{H}$ diagram due to larger Joule heating in $\mathrm{P}$ state (low resistance) than in AP state (high resistance).

In FIG. 1(c), the sample has a very low TMR amplitude, so the mechanism of asymmetric heating described above is negligible. But one can still see a small difference in switching voltage: $V_{\mathrm{sw}}^{+}=0.32 \mathrm{~V}, V_{\mathrm{sw}}^{-}=0.34 \mathrm{~V}$. This polarity dependence in the shape of $\mathrm{V}-\mathrm{H}$ diagram might originate from the heating asymmetry induced by tunneling current [25]. Most of Joule energy is released after the electrons tunneling in the receiving magnetic electrode adjacent to the $\mathrm{MgO}$ barrier. Therefore, considering that the thermal conductivity of thin $\mathrm{MgO}$ barrier is much lower than that of bulk $\mathrm{MgO}$ [21], the storage layer reaches up to $10 \%$ higher temperature if the current polarity is such that the storage layer receives the tunneling electrons. This effect becomes more significant in the case of $\mathrm{MgO}$-based capping layer since the storage layer appears to be thermally insulated between two $\mathrm{MgO}$ layers with low thermal conductivity.

Another source of asymmetry in the $\mathrm{V}-\mathrm{H}$ diagram can result from the voltage dependence of the torque amplitude associated with elastic tunneling in MTJ [26]. Indeed, the parallel component of the torque $T_{\|}$varies as $T_{\|} \sim a_{1} V+a_{2} V^{2}$. The transport parameter $a_{2}$ is known to vanish for MTJs with ferromagnetic electrodes having large spin splitting or being half-metallic. Such MTJs are expected to exhibit high TMR amplitude. In such MTJs, if heating effects are not taken into account, the switching voltages for P-AP and AP-P transitions are therefore close to opposite $\left(V_{\mathrm{sw}}^{+}=V_{\mathrm{sw}}^{-}\right)$. On the other hand, for low TMR MTJs, the parallel torque exhibits a nonsymmetric quadratic voltage behavior so that switching 
voltages do not coincide $\left(V_{\mathrm{sw}}^{+} \neq V_{\mathrm{sw}}^{-}\right)$.

To get deeper insight on the above mentioned features of the experimental V-H diagrams, we developed a model accounting for the heating effects in the storage layer. The standard phenomenological macrospin-based model was modified by including a dependence of the storage layer temperature on voltage and a resulting dependence of magnetization, spin polarization and anisotropy constant on the applied voltage pulse.

\section{MODEL}

The first adjustment of the model concerns the change in anisotropy energy while changing the bias voltage. There are several possible mechanisms affecting the uniaxial anisotropy constant. A first one is a direct voltage induced variation of anisotropy due to interfacial charge modulation [27-30], but this effect is relatively weak compared to the influence of the temperature variation associated with Joule heating. A second mechanism - is a temperature induced variation of the anisotropy due to Joule heating, which is the most significant for our samples. Concerning other thermoelectric effects, as it was shown by Flipse et al [31] and later by Avery and Zink [32] the Peltier coefficient is of order $\Pi \sim 10^{-3} \mathrm{~V}$ and thus the Peltier power at $R \sim 10^{3} \Omega$ and $V \sim 1 \mathrm{~V}$ is $Q_{\Pi} \sim \Pi \cdot V / R \sim 10^{-6} \mathrm{~W}$, while the Joule power $Q_{J}=V^{2} / R \sim 10^{-3} \mathrm{~W}$ is larger by three orders of magnitude. Seebeck effect is also negligible as its coefficient $S$ is expressed through $\Pi$ by Thomson-Onsager relation $\Pi=S \cdot T$.

We first estimated the temperature dependence on the bias voltage in MTJ starting from the first law of thermodynamics and describing the MTJ with a simple one dimensional model according to the following differential equation:

$$
\frac{V^{2}}{R}-Q\left(T-T_{0}\right)=C \frac{d T}{d t},
$$

where $V^{2} / R$ is a Joule power, $R$ - resistance, $Q$ is the heat transfer coefficient between the tunnel barrier and the conductive bottom and top electrodes assumed to be at a fixed temperature $T_{0}, T_{0}$ is the room temperature and $C$ is the MTJ heat capacity. Equation (1) gives a solution for the temperature $T$ as a function of pulse duration $t_{p}$ :

$$
T=T_{0}+\frac{V^{2}}{R} Q\left(1-\mathrm{e}^{-(Q / C) t_{p}}\right) .
$$

We assume that the temperature of the storage layer follows this square dependence on voltage pulse amplitude (2) and all other parameters are kept constant for a given sample. Then, a second step in the model development consists in assuming that the temperature dependence of the uniaxial anisotropy constant is follows the
Calen-Calen law [33]:

$$
K(T)=K_{0}\left(\frac{M_{s}(T)}{M_{s 0}}\right)^{\xi}
$$

where $K_{0}$ is the uniaxial anisotropy constant at zero temperature, $M_{s 0}$ is the spontaneous magnetization of the storage layer at zero temperature. From previous studies, the value of the exponent $\xi$ was experimentally estimated to lie in the range between 2 and 3 [20, 34-37]. The temperature dependence of the storage layer magnetization is supposed to follow a Bloch law:

$$
M_{s}(T)=M_{s 0}\left(1-\left(T / T_{c}\right)^{3 / 2}\right)
$$

where $T_{c}$ is the Curie temperature of the storage layer. The exponent " $3 / 2$ " is slightly different for thin deposited films, and its experimentally determined value equals to 1.73 [34]. Taking into account (3) and (4) and assuming that pulse durations are long enough so that the temperature varies with the voltage as $T=T_{0}+k_{V} V^{2}$ according to $(2)$, the voltage dependences of the magnetization and anisotropy constant reads:

$$
\begin{gathered}
M_{s}(V)=M_{s 0}\left(1-\left(\frac{T_{0}+k_{V} V^{2}}{T_{c}}\right)^{1.73}\right) \\
K(V)=K_{0}\left(1-\left(\frac{T_{0}+k_{V} V^{2}}{T_{c}}\right)^{1.73}\right)^{\xi},
\end{gathered}
$$

where $k_{V}$ is a parameter dependent on the writing pulse duration, the thermal capacity of the storage layer, electrical resistance and the thermal resistance of the stack around the storage layer. The parameter $k_{V}$ depends also on the polarity of the applied voltage as mentioned earlier [25]. Actually in the following of our model, the heating asymmetry was not taken into account and $k_{V}$ was kept independent on current polarity.

We want to underline that in case of pulsed $\mathrm{V}-\mathrm{H}$ diagram, the observed back switching anomalies are not only caused by the voltage induced anisotropy reduction but also by the reduction of spin polarization. As the anisotropy amplitude is restored at the end of the voltage pulse, the fact that the storage layer had time to switch or not during the pulse has a great importance. If STT efficiency becomes too weak due to the heating, the storage layer may not be able to switch any more. This is the reason why we can cross the critical lines twice on V-H diagram at a given applied field (e.g. FIG. 1(c) increase voltage at 150 Oe from $0 \mathrm{~V}$ to $0.6 \mathrm{~V}$ ).

The phenomenological macrospin model used here is the same as described in Ref. [11, 13] but it takes into account the voltage dependence of several parameters. Landau-Lifshitz-Gilbert (LLG) equation for the magne- 
tization dynamics is written as:

$$
\begin{aligned}
& \frac{d \mathbf{m}}{d t}=-\gamma\left(\mathbf{m} \times \mu_{0} \mathbf{H}_{\mathrm{eff}}(V)\right)+\alpha\left(\mathbf{m} \times \frac{d \mathbf{m}}{d t}\right) \\
& -\gamma a_{\|}(V) V[\mathbf{m} \times(\mathbf{m} \times \mathbf{p})]+\gamma a_{\perp}(V) V^{2}(\mathbf{m} \times \mathbf{p}),
\end{aligned}
$$

where $\mathbf{m}$ is the unit vector of the magnetization of the storage layer, $\alpha$ is the Gilbert damping factor, $\gamma$ is the gyromagnetic ratio of free electrons, $\mathbf{p}$ is the unit vector along the spin current polarization (magnetization of the reference layer), $a_{\|}(V)$ and $a_{\perp}(V)$ are phenomenological transport parameters depending on the applied voltage. The effective field $\mathbf{H}_{\text {eff }}(V)$ in our model depends on applied voltage and derives from the Gibbs free energy $E(V)$ density functional:

$$
\begin{aligned}
E(V) & =-K(V)\left(\mathbf{u}_{K} \cdot \mathbf{m}\right)^{2}-\mu_{0} M_{s}(V) \mathbf{H}_{\mathrm{ext}}(V) \cdot \mathbf{m} \\
& +\frac{1}{2} \mu_{0} M_{s}^{2}(V)\left(N_{x} m_{x}^{2}+N_{y} m_{y}^{2}+N_{z} m_{z}^{2}\right),
\end{aligned}
$$

where $\mathbf{H}_{\text {ext }}$ is the external applied field, $\mathbf{u}_{K}$ is the unit vector along the easy axis (here $\mathrm{Oz}$ ), $N_{x, y, z}$ are diagonal terms of the demagnetizing tensor, $M_{s}(V)$ and $K(V)$ are defined from (5). The first term in (7) represents the anisotropy energy $E_{K}(V)$, so the corresponding effective anisotropy field reads:

$$
\begin{aligned}
H_{K}(V) & =\frac{2 K(V)}{\mu_{0} M_{s}(V)} \\
& =H_{K 0}\left(1-\left(\frac{T_{0}+k_{V} V^{2}}{T_{c}}\right)^{1.73}\right)^{\xi},
\end{aligned}
$$

where $H_{K 0}=2 K_{0} /\left(\mu_{0} M_{s 0}\right)$. The dependence of normalized effective anisotropy field (8) on applied voltage for different values of exponent $\xi$ is presented in FIG. 4. In the inset, the same dependence is plotted versus the temperature reached by the storage layer during the voltage pulse $T=T_{0}+k_{V} V^{2}$. This dependence is almost linear as it was shown in the experiment [34, 38]. Assuming the ground state of storage layer to be out-of-plane, the field-like torque term $\sim a_{\perp}(V)$ in (6) is neglected here since the Slonczewski term $\sim a_{\|}(V)$ is known to play a dominant role in the switching process. The phenomenological transport parameter $a_{\|}(V)$ is proportional to the spin polarization which follows the magnetization variation given by (5) (see solid black line in FIG. 4) as reported in Ref. [19]. Consequently, due to the Joule heating, the STT prefactor has the following dependence on voltage:

$$
a_{\|}(V)=a_{\| 0}\left(1-\left(\frac{T_{0}+k_{V} V^{2}}{T_{c}}\right)^{1.73}\right),
$$

where $a_{\| 0}$ represents the transport parameter value at zero temperature.

Having set the model as described above, the numerical switching V-H diagrams are computed following the experimental procedure. The magnetization of the storage

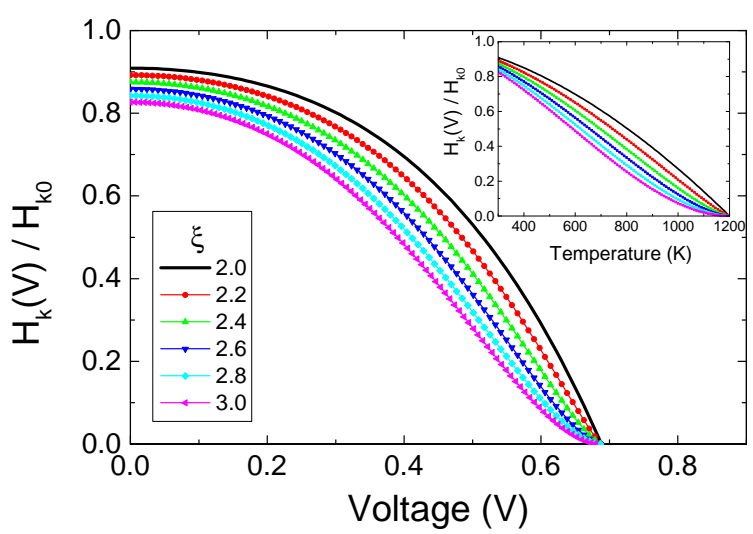

FIG. 4. Normalized effective anisotropy field versus applied voltage according to (8) for different values of the exponent $\xi$ using the parameters: $T_{0}=300 \mathrm{~K}, k_{V}=1900 \mathrm{~K} / \mathrm{V}^{2}$, $T_{c}=1200 \mathrm{~K}$. The curve for $\xi=2$ (solid black line) repeats the normalized magnetization $M_{s}(V) / M_{s 0}$. In the inset the same function versus resulting temperature $T=T_{0}+k_{V} V^{2}$.

layer is relaxed under the given applied field. After that a writing pulse is applied for $100 \mathrm{~ns}$. The total integration time is $1 \mu \mathrm{s}$. The amplitude of applied field is changed from its minimum to maximum value and vice versa to reproduce a hysteresis loop. The initial room temperature value was set at $T_{0}=300 \mathrm{~K}$. The thermal fluctuations are described by random field added to the total effective field as a white noise field with the following properties:

$$
\begin{aligned}
\left\langle\mathbf{H}_{\mathrm{TR}}(t)\right\rangle & =0, \\
\left\langle\mathbf{H}_{\mathrm{TR}}(t) \mathbf{H}_{\mathrm{TR}}\left(t^{\prime}\right)\right\rangle & =\frac{2 \alpha k_{B} T}{\gamma \mu_{0} M_{s} \Omega} \delta\left(t-t^{\prime}\right),
\end{aligned}
$$

where $k_{B}$ is the Boltzmann constant and $\Omega$ is the sample volume. Each hysteresis loop at fixed voltage was repeated 10 times resulting an averaged $\mathrm{V}-\mathrm{H}$ loop similar to the experimental diagram.

The numerical V-H diagrams computed for a disk of $100 \mathrm{~nm}$ diameter and $1.6 \mathrm{~nm}$ thick are presented in FIG. 5 for several values of the exponent $\xi$. The shape of the diagram evolves progressively with the value of the exponent $\xi$. Two regimes are identified. At low values of $\xi$ (around 2 ), the numerical diagram is very similar to the experimental diagram plotted in FIG. 1(d). In contrast, when $\xi$ approaches the upper limit of 3 , a strong similarity with the experimental diagram FIG. 1(b,c) is observed. In this second regime, two specific critical lines are identified: a first one, STT driven, at low voltages and a second one, heat driven, at high voltages. The parameter $\xi$ accounts for the strength of the dependence of the material parameters on the voltage. The impact of its value on the shape of the $\mathrm{V}-\mathrm{H}$ diagrams is quite drastic, confirming the important role of Joule heating on switching abilities of MTJs. To better understand the underlying parameters behind these critical lines and eventually find how 
to control them, we developed an analytical model based on the stability analysis of the linearized LLG equation.

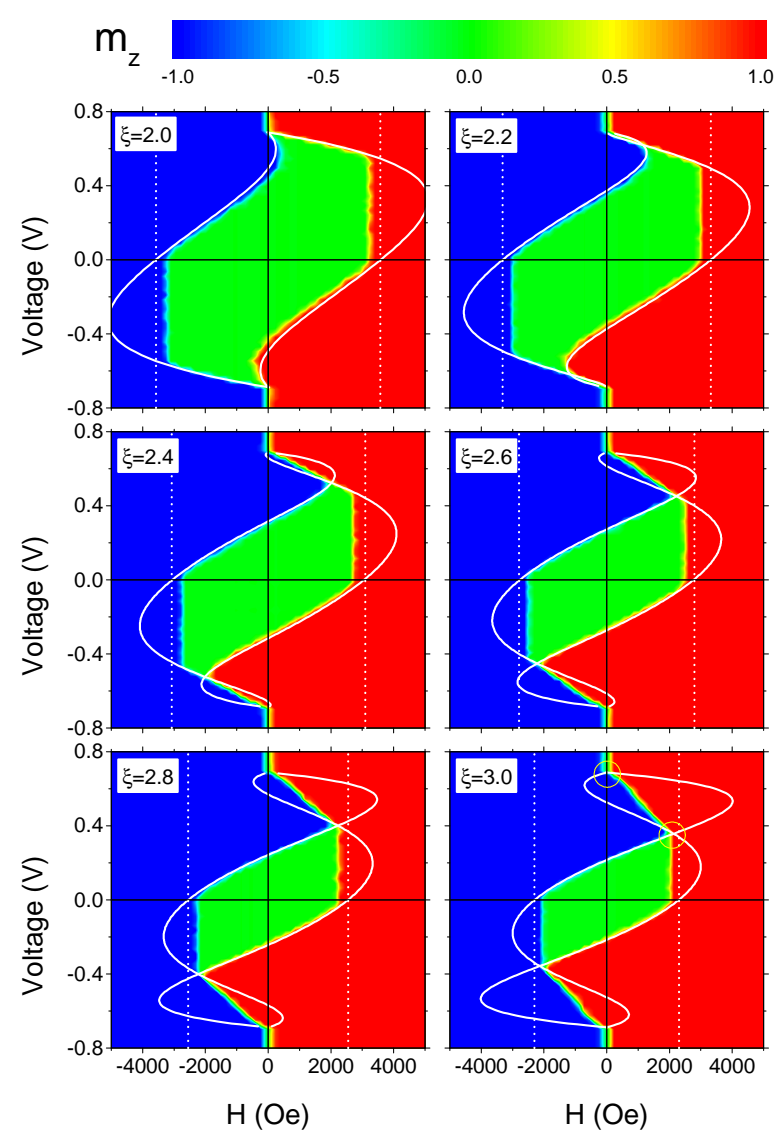

FIG. 5. Numerical stability V-H diagrams for different values of exponent $\xi$ at room temperature $T_{0}=300 \mathrm{~K}, k_{V}=1900 \mathrm{~K} / \mathrm{V}^{2}, T_{c}=1200 \mathrm{~K}, M_{s 0}=10^{6} \mathrm{~A} / \mathrm{m}$, $K_{0}=778319 \mathrm{~J} / \mathrm{m}^{3}, \alpha=0.01, a_{\perp 0}=0, a_{\| 0}=8 \cdot 10^{-3} \mathrm{~T} / \mathrm{V}, \mathbf{p}=(0$, $0,-1), N_{x}=N_{y}=0.025, N_{z}=0.95$ using 10 averaged loops for each voltage with resolution $64 \times 64$ points. Critical lines (white solid curves) calculated from equations (18) and superposed with the numerical V-H diagrams. Vertical dashed lines correspond to the coercive field calculated from the solution of the equations (17) at zero applied voltage. For $\xi=3.0$ the intersection points of two solutions are circled.

\section{ANALYTICAL MODEL}

The critical lines of the numerically obtained V-H diagrams presented in FIG. 4 (white lines) can be extracted analytically at zero temperature using the generic model of a nonlinear auto-oscillator proposed by Slavin and Tiberkevich [39]. Following the Holstein-Primakoff transformation the components of the unitary magnetization vector $\mathbf{m}$ in (6) are replaced by the canonical variables $c$ and $c^{*}$ as proposed in Ref. [40]:

$$
c=\frac{m_{x}-\imath m_{y}}{\sqrt{2\left(1+m_{z}\right)}} .
$$

It is convenient to express the modified LLG equation (6) in complex variables with four terms: precession, damping, field-like and damping-like:

$$
\frac{d c}{d t}=\left.\frac{d c}{d t}\right|_{\mathrm{prec}}+\left.\frac{d c}{d t}\right|_{\mathrm{damp}}+\left.\frac{d c}{d t}\right|_{\mathrm{FL}}+\left.\frac{d c}{d t}\right|_{\mathrm{DL}}
$$

Assuming that the polarization vector $\mathbf{p}=\left(0,0, p_{z}\right)$ and the applied field $\mathbf{H}_{\text {ext }}=\left(0,0, H_{z}\right)$ are both perfectly out-ofplane, the full expression of each term is explicitly given below:

$$
\begin{aligned}
\left.\frac{d c}{d t}\right|_{\text {prec }} & =-\imath c\left[\omega_{H}+\left(\omega_{M}-\omega_{A}\right)\left(2|c|^{2}-1\right)\right], \\
\left.\frac{d c}{d t}\right|_{\text {damp }} & =-\alpha c\left(1-|c|^{2}\right) \times \\
\left.\frac{d c}{d t}\right|_{\mathrm{FL}} & =\imath\left(\alpha \omega_{\|}+\omega_{\perp}\right) p_{z} c \\
\left.\frac{d c}{d t}\right|_{\mathrm{DL}} & =-\left(\omega_{\|}-\alpha \omega_{\perp}\right)\left(1-|c|^{2}\right) p_{z} c
\end{aligned}
$$

where

$$
\begin{gathered}
\omega_{H}=\frac{\gamma}{1+\alpha^{2}} H_{z}, \quad \omega_{A}=\frac{\gamma}{1+\alpha^{2}} \frac{2 K_{u}}{\mu_{0} M_{s}} \\
\omega_{\|}=\frac{\gamma}{1+\alpha^{2}} \frac{a_{\|} V}{\mu_{0}}, \quad \omega_{\perp}=\frac{\gamma}{1+\alpha^{2}} \frac{a_{\perp} V^{2}}{\mu_{0}} \\
\omega_{M}=\frac{\gamma}{1+\alpha^{2}}\left(N_{z}-N_{x}\right) M_{s} .
\end{gathered}
$$

Since the complex variable $c$ has an amplitude $p$ and a phase $\phi$ associated by the relation $c(t)=\sqrt{p(t)} \mathrm{e}^{\phi(t)}$, one can then derive two equations:

$$
\begin{gathered}
\frac{d p}{d t}=-2 \alpha\left[\omega_{H}+\left(\omega_{\|} / \alpha-\omega_{\perp}\right) p_{z}+\right. \\
\left.\left(\omega_{M}-\omega_{A}\right)(2 p-1)\right](p-1) p \\
\frac{d \phi}{d t}=-\left[\begin{array}{c}
\omega_{H}-\left(\alpha \omega_{\|}+\omega_{\perp}\right) p_{z}+ \\
\left.\left(\omega_{M}-\omega_{A}\right)(2 p-1)\right] .
\end{array}\right.
\end{gathered}
$$

Looking for the stationary solutions $d p / d t=0$, two static solutions are found: i) $p_{0}=0$ meaning magnetization "up" $m_{z}=+1$ and ii) $p_{0}=1$ meaning magnetization "down" $m_{z}=-1$.

By linearizing the equation $d p / d t$ in (15) around the solution $p_{0}$ and keeping only the first order terms, one 
can derive an equation for the power deviation $\delta p$ :

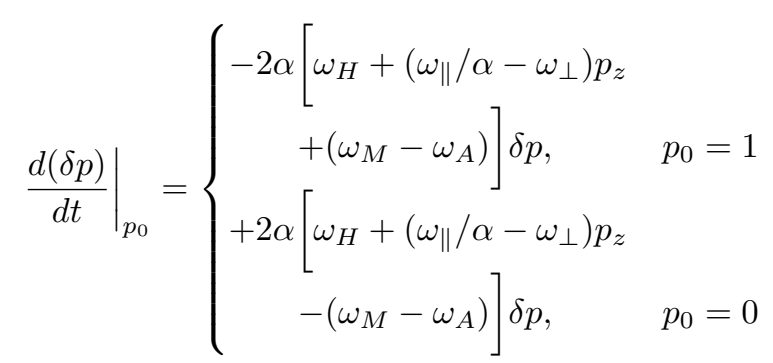

If the pre-factor in front of $\delta p$ becomes positive, the deviation $\delta p$ will diverge and the static equilibrium becomes unstable. From this condition, by setting the pre-factors to zero, one can derive the boundaries of stability under static field and/or voltage. Two critical lines for the applied field without any applied voltage are given by:

$$
\begin{aligned}
& \omega_{H}+\left(\omega_{M}-\omega_{A}\right)=0, \\
& \omega_{H}-\left(\omega_{M}-\omega_{A}\right)=0 .
\end{aligned}
$$

The two critical lines for dc field and voltage are:

$$
\begin{aligned}
& \omega_{H}+\left(\omega_{\|} / \alpha-\omega_{\perp}\right) p_{z}+\left(\omega_{M}-\omega_{A}\right)=0 \\
& \omega_{H}+\left(\omega_{\|} / \alpha-\omega_{\perp}\right) p_{z}-\left(\omega_{M}-\omega_{A}\right)=0 .
\end{aligned}
$$

Substituting $M_{s}, K_{u}$ and $a_{\|}$in (14) by the corresponding functions of the bias voltage given by expressions (5) and (9), the solution of equations (17) and (18) gives critical the curves $H(V)$ which coincide well with the boundaries of the numerical diagrams. In FIG. 5, the critical lines are superposed to the numerical $\mathrm{V}-\mathrm{H}$ diagrams. Vertical dashed lines correspond to the coercive field calculated from the solution of equations (17) at zero applied voltage, which correspond to a finite pulse duration. If the voltage pulse is infinite $(\mathrm{dc})$ the solution (17) reproduces the boundaries like in FIG. 2(a) - coercive field decreases with applied bias voltage. The small difference between the numerical and analytical coercive field is due to the fluctuating term (10). It increases the probability of magnetization switching before the field reaches its coercive value and yields this $10 \%$ deviation. The critical lines under dc field and dc voltage are rather complex and partially recover the numerical boundaries at low $\xi$ and low voltage pulses. At $\xi$ value close to 3 , the critical lines at high voltages are closer to that in the dc diagram from FIG. 2(a). The analytical dc curves reproduce well the two intersection points through which the numerical critical lines pass.

In the case where $\xi$ equals 2 or 3 and the magnetization follows the Bloch law (4), one can find the solution for intersection points of the critical lines defined by equations (18) (see yellow circles in FIG. 5 for $\xi=3.0$ ). Considering that the magnetization depends on voltage due to Joule heating and introducing the function $f_{B}(V)$ in (5) defined by $f_{B}(V)=M_{s}(V) / M_{s 0}$, neglecting the field-like torque term $\omega_{\perp}=0$ and assuming the polarization is "up" $p_{z}=1$, one gets the following equations from (14) and (18):

$$
\begin{aligned}
H_{z}+ & \frac{a_{\| 0} V}{\mu_{0} \alpha} f_{B}(V)+ \\
& {\left[M_{s 0}\left(N_{z}-N_{x}\right) f_{B}(V)-H_{K 0} f_{B}^{\xi-1}(V)\right]=0, } \\
H_{z}+ & \frac{a_{\| 0} V}{\mu_{0} \alpha} f_{B}(V)- \\
& {\left[M_{s 0}\left(N_{z}-N_{x}\right) f_{B}(V)-H_{K 0} f_{B}^{\xi-1}(V)\right]=0, }
\end{aligned}
$$

where $H_{K 0}=2 K_{0} /\left(\mu_{0} M_{s 0}\right)$. Now it is clear that to satisfy these equations (19) at the same value of applied field $H_{z}$, the last term in brackets should be equal to zero. If the exponent $\xi=2$, then the solution is trivial: $f_{B}(V)=0$. The intersection points correspond to heating the storage layer up to the Curie temperature: $V_{c 0}=$ $\pm \sqrt{\left(T_{c}-T_{0}\right) / k_{V}}$. If the exponent $\xi=3$, then besides the trivial solution, there is another one which reads:

$$
V_{c 1}= \pm \sqrt{\frac{T_{c}}{k_{V}}} \sqrt{(1-\eta)^{2 / 3}-\frac{T_{0}}{T_{c}}}
$$

where $\eta=M_{s 0}\left(N_{z}-N_{x}\right) / H_{K 0}$. As one can see, the ratio $V_{c 0} / V_{c 1}$ depends on the factor $\eta$ and Curie temperature $T_{c}$. The thermally induced slope that passes through these intersection points also depends on these parameters but does not dependent on the heating coefficient $k_{V}$ :

$$
\begin{aligned}
\left.\frac{d V}{d H}\right|_{\xi=3}= & \frac{V_{c 1}-V_{c 0}}{H_{c 1}}= \\
& -\frac{1}{\eta} \frac{\mu_{0} \alpha}{a_{\| 0}}\left(1-\frac{\sqrt{1-T_{0} / T_{c}}}{\sqrt{(1-\eta)^{2 / 3}-T_{0} / T_{c}}}\right) .
\end{aligned}
$$

Therefore, this slope should not depend on the voltage pulse duration as it does not depend on $k_{V}$. On the other hand, if the exponent $\xi=2$, the thermally induced slope can be calculated as an inverted derivative of the function $H_{z}(V)$ extracted from (19) taken at the point $V=V_{c 0}$ :

$$
\begin{aligned}
& \left.\frac{d V}{d H}\right|_{\xi=2}=\left(\left.\frac{d H}{d V}\right|_{V=V_{c 0}}\right)^{-1}= \\
& \quad-\frac{1}{3}\left[\frac{a_{\| 0}}{\mu_{0} \alpha}\left(1-\frac{T_{0}}{T_{c}}\right)+\right. \\
& \left.M_{s 0}\left(N_{z}-N_{x}\right) \frac{1-\eta}{\eta} \sqrt{\frac{k_{V}}{T_{c}}} \sqrt{1-T_{0} / T_{c}}\right]^{-1} .
\end{aligned}
$$

The slope slightly increases with decreasing writing pulse duration. In FIG. 6, the critical lines extracted from the experimental diagrams for different values of the writing pulse duration are presented. The switching voltage is almost constant at pulse durations more than $100 \mathrm{~ns}$ and increases sharply for shorter pulses as it was observed earlier [13]. The thermally induced boundaries 
remain almost constant at pulses longer than 30 ns and moves upward for shorter pulses with slight increase of the slope.

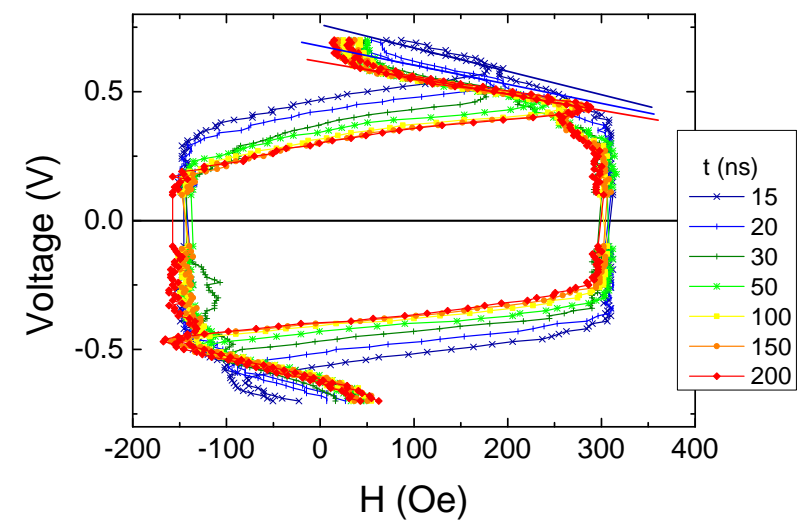

FIG. 6. Critical lines extracted from the experimental diagrams of the sample from FIG. 1(c) for different lengths of applied voltage pulse. Thermally induced boundaries remain almost constant at pulses longer than 30 ns and move upwards for shorter pulses with slight increase of the slope. See sample details in TAB. I.

The macrospin model used in this study gives an overestimate value for the coercive field which can be found from equations (17) at zero applied voltage:

$$
\begin{aligned}
H_{c}= & \pm M_{s 0}\left(N_{z}-N_{x}\right) \times \\
& \left(1-\left(\frac{T_{0}}{T_{c}}\right)^{x}\right)\left[\frac{1}{\eta}\left(1-\left(\frac{T_{0}}{T_{c}}\right)^{x}\right)^{\xi-2}-1\right] .
\end{aligned}
$$

For the FeCoB-based storage layer with a diameter of $100 \mathrm{~nm}$, theoretical $H_{c}$ in macrospin approximation should be of the order of $\sim 1 \mathrm{~T}\left(10^{4} \mathrm{Oe}\right)$. But in the experiment, its value is usually ten times smaller $\sim 0.1 \mathrm{~T}\left(10^{3} \mathrm{Oe}\right)$ due to the non-uniform switching mode (not macrospin). Similarly, the STT switching is not macrospin for the used MTJ diameters. Therefore, to fit the experimental diagrams with this model, one has to use an effective value for the uniaxial anisotropy constant to account for the fact that the barrier height for switching is different from that of macrospin uniform switching. The result of the fitting is presented in FIG. 7. The first sample in FIG. 7(a) has a low TMR value and the critical lines are almost symmetric. FIG. 7(b) shows a diagram of the sample from the same wafer as the sample in FIG. 1(b) measured under finite voltage pulse. As the TMR amplitude is larger than for the sample of FIG. 7(a), the asymmetry of STT boundaries is more pronounced; the STT boundary corresponding to the PAP transition is shifted towards the zero voltage, because more heat is produced in the low resistance state $(\mathrm{P})$ in the storage layer which reduces the switching voltage. To take into account the heating asymmetry in our model,
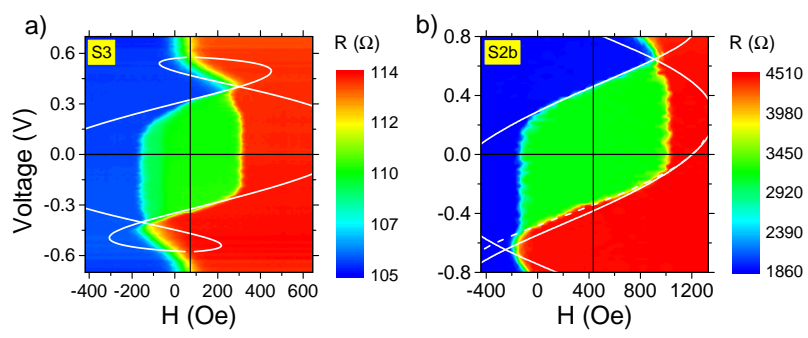

FIG. 7. Experimental diagrams fit with the analytical solution. (a) Sample from FIG. 1(c) with low TMR, $\xi=2.16$, $M_{s 0}=900 \mathrm{kA} / \mathrm{m}, K_{0}=570 \mathrm{~kJ} / \mathrm{m}^{3}, k_{V}=1200 \mathrm{~K} / \mathrm{V}^{2}, T_{c}=700 \mathrm{~K}$, $a_{\| 0}=1.4 \mathrm{mT} / \mathrm{V}, N_{x}=N_{y}=0.01, N_{z}=0.98$. Other parameters the same as in FIG. 5. (b) Sample from the same wafer as the sample in FIG. 1(b) with high $\mathrm{TMR}=142 \%, \xi=2.3$, $M_{s 0}=900 \mathrm{kA} / \mathrm{m}, K_{0}=580 \mathrm{~kJ} / \mathrm{m}^{3}, k_{V}=340 \mathrm{~K} / \mathrm{V}^{2}, T_{c}=700 \mathrm{~K}$, $a_{\| 0}=2 \mathrm{mT} / \mathrm{V}$. Dashed line corresponds to the critical curve at $k_{V}=500 \mathrm{~K} / \mathrm{V}^{2}$. Other parameters the same as in FIG. 5.

one can just take different $k_{V}$ value for AP-P and $\mathrm{P}$ AP critical lines. To roughly estimate the dependence of the asymmetry factor $\zeta=\left(V_{c 0}^{A P \rightarrow P}-V_{c 0}^{P \rightarrow A P}\right) / V_{c 0}^{P \rightarrow A P}$ on TMR value, assuming that $k_{V}$ depends only on resistance of MTJ (2), one can use the expression $\zeta=$ $\sqrt{R_{A P} / R_{P}}-1=\sqrt{1+T M R}-1$.

\section{SUMMARY}

To conclude, we measured the stability V-H diagrams in a variety of samples of different composition and diameters in which significant Joule heating take place during STT writing. We explained the high voltage anomaly of the $\mathrm{V}-\mathrm{H}$ diagrams as due to heating effects in the storage layer. Two mechanisms of the asymmetry of the critical lines for positive and negative voltages were suggested: heating asymmetry induced by the asymmetric inelastic relaxation of tunneling electrons and heating asymmetry due to the difference of the resistance in $\mathrm{P}$ and $\mathrm{AP}$ states. The second mechanism makes the largest contribution to the heating asymmetry versus bias voltage when the TMR is large. We also developed a modified macrospin-based model by including a dependence of the material parameters on the applied voltage, such as uniaxial anisotropy constant $\left(K_{u}\right)$, spontaneous magnetization $\left(M_{s}\right)$ and STT prefactor $a_{\|}$. The model was used to numerically simulate the V-H diagrams. Good agreement with the experimental results was obtained. Furthermore, we derived explicit analytical expressions for the critical lines that exactly follow the contours of the numerical diagram. The obtained expressions can be applied to fit also the experimental diagrams, but some effective values for $K_{u}$ and $M_{s}$ should be used, as the switching of the real samples occurs in a non-uniform regime (for example, by domain wall propagation) which reduces the coercive field. 
The presented model can be used in pMTJ-based MRAM circuits design to take into account such unusual behavior of the MTJ properties at high bias voltages. Moreover, the predictions presented in this study can be extrapolated to similar multilayer stacks used for other applications.

Acknowledgements: This work was supported by ERC Advanced Grant MAGICAL n ${ }^{\circ} 669204$.

* nik@magn.phys.msu.ru

† bernard.dieny@cea.fr

[1] J. Slonczewski, Journal of Magnetism and Magnetic Materials 159, L1 (1996), arXiv:arXiv:1601.07392v2.

[2] L. Berger, Physical Review B 54, 9353 (1996), arXiv:1102.5325.

[3] H. Yoda, T. Kishi, T. Nagase, M. Yoshikawa, K. Nishiyama, E. Kitagawa, T. Daibou, M. Amano, N. Shimomura, S. Takahashi, T. Kai, M. Nakayama, H. Aikawa, S. Ikegawa, M. Nagamine, J. Ozeki, S. Mizukami, M. Oogane, Y. Ando, S. Yuasa, K. Yakushiji, H. Kubota, Y. Suzuki, Y. Nakatani, T. Miyazaki, and K. Ando, Current Applied Physics 10, e87 (2010).

[4] S. Ikeda, K. Miura, H. Yamamoto, K. Mizunuma, H. D. Gan, M. Endo, S. Kanai, J. Hayakawa, F. Matsukura, and H. Ohno, Nature Materials 9, 721 (2010), arXiv:NIHMS150003.

[5] N. Tezuka, S. Oikawa, I. Abe, M. Matsuura, S. Sugimoto, K. Nishimura, and T. Seino, IEEE Magnetics Letters 7, 1 (2016).

[6] M. Wang, W. Cai, K. Cao, J. Zhou, J. Wrona, S. Peng, H. Yang, J. Wei, W. Kang, Y. Zhang, J. Langer, B. Ocker, A. Fert, and W. Zhao, Nature Communications 9, 671 (2018).

[7] H. Sato, E. C. Enobio, M. Yamanouchi, S. Ikeda, S. Fukami, S. Kanai, F. Matsukura, and H. Ohno, Applied Physics Letters 105, 2012 (2014).

[8] S.-E. Lee, Y. Takemura, and J.-G. Park, Applied Physics Letters 109, 182405 (2016).

[9] D. C. Worledge, G. Hu, D. W. Abraham, J. Z. Sun, P. L. Trouilloud, J. Nowak, S. Brown, M. C. Gaidis, E. J. O'Sullivan, and R. P. Robertazzi, Applied Physics Letters 98, 22501 (2011).

[10] K. Bernert, V. Sluka, C. Fowley, J. Lindner, J. Fassbender, and A. M. Deac, Physical Review B - Condensed Matter and Materials Physics 89, 134415 (2014).

[11] A. A. Timopheev, R. Sousa, M. Chshiev, L. D. BudaPrejbeanu, and B. Dieny, Physical Review B - Condensed Matter and Materials Physics 92, 104430 (2015), arXiv:1506.0780.

[12] W. Skowroński, M. Czapkiewicz, S. Ziętek, J. Chęciński, M. Frankowski, P. Rzeszut, and J. Wrona, Scientific Reports 7, 10172 (2017).

[13] N. Strelkov, A. Timopheev, R. C. Sousa, M. Chshiev, L. D. Buda-Prejbeanu, and B. Dieny, Physical Review B 95, 184409 (2017).

[14] P. Gaunt, Philosophical Magazine B 48, 261 (1983).

[15] W. C. Nunes, W. S. D. Folly, J. P. Sinnecker, and M. A. Novak, Physical Review B 70, 014419 (2004).
[16] M. Laufenberg, W. Bührer, D. Bedau, P.-E. Melchy, M. Kläui, L. Vila, G. Faini, C. A. F. Vaz, J. A. C. Bland, and U. Rüdiger, Physical Review Letters 97, 046602 (2006).

[17] K. Ueda, T. Koyama, R. Hiramatsu, D. Chiba, S. Fukami, H. Tanigawa, T. Suzuki, N. Ohshima, N. Ishiwata, Y. Nakatani, K. Kobayashi, and T. Ono, Applied Physics Letters 100, 202407 (2012).

[18] E. Villamor, M. Isasa, L. E. Hueso, and F. Casanova, Physical Review B 88, 184411 (2013).

[19] V. Garcia, M. Bibes, A. Barthélémy, M. Bowen, E. Jacquet, J.-P. Contour, and A. Fert, Physical Review B 69, 052403 (2004).

[20] S. Bandiera, R. C. Sousa, M. Marins de Castro, C. Ducruet, C. Portemont, S. Auffret, L. Vila, I. L. Prejbeanu, B. Rodmacq, and B. Dieny, Applied Physics Letters 99, 202507 (2011).

[21] T. Böhnert, R. Dutra, R. L. Sommer, E. Paz, S. SerranoGuisan, R. Ferreira, and P. P. Freitas, Physical Review B 95, 104441 (2017).

[22] V. D. Nguyen, P. Sabon, J. Chatterjee, L. Tille, P. V. Coelho, S. Auffret, R. Sousa, L. Prejbeanu, E. Gautier, L. Vila, and B. Dieny, in 2017 IEEE International Electron Devices Meeting (IEDM) (2017) pp. 38.5.1-38.5.4.

[23] J. Chatterjee, R. C. Sousa, N. Perrissin, S. Auffret, C. Ducruet, and B. Dieny, Applied Physics Letters 110, 202401 (2017).

[24] A. Chavent, C. Ducruet, C. Portemont, L. Vila, J. Alvarez-Hérault, R. Sousa, I. L. Prejbeanu, and B. Dieny, Physical Review Applied 6, 034003 (2016).

[25] E. Gapihan, J. Hérault, R. C. Sousa, Y. Dahmane, B. Dieny, L. Vila, I. L. Prejbeanu, C. Ducruet, C. Portemont, K. Mackay, and J. P. Nozières, Applied Physics Letters 100, 202410 (2012).

[26] M. Chshiev, A. Manchon, A. Kalitsov, N. Ryzhanova, A. Vedyayev, N. Strelkov, W. H. Butler, and B. Dieny, Physical Review B - Condensed Matter and Materials Physics 92, 104422 (2015), arXiv:1308.2619.

[27] T. Nozaki, Y. Shiota, M. Shiraishi, T. Shinjo, and Y. Suzuki, Applied Physics Letters 96, 022506 (2010).

[28] Y. Shiota, S. Murakami, F. Bonell, T. Nozaki, T. Shinjo, and Y. Suzuki, Applied Physics Express 4, 43005 (2011).

[29] T. Nozaki, H. Arai, K. Yakushiji, S. Tamaru, H. Kubota, H. Imamura, A. Fukushima, and S. Yuasa, Applied Physics Express 7, 73002 (2014).

[30] Z. Wen, H. Sukegawa, T. Seki, T. Kubota, K. Takanashi, and S. Mitani, Scientific Reports 7, 45026 (2017).

[31] J. Flipse, F. L. Bakker, A. Slachter, F. K. Dejene, and B. J. van Wees, Nature Nanotechnology 7, 166 (2012).

[32] A. D. Avery and B. L. Zink, Physical Review Letters 111, 126602 (2013).

[33] H. B. Callen and E. Callen, Journal of Physics and Chemistry of Solids 27, 1271 (1966).

[34] K. M. Lee, J. W. Choi, J. Sok, and B. C. Min, AIP Advances 7, 065107 (2017).

[35] J. G. Alzate, P. Khalili Amiri, G. Yu, P. Upadhyaya, J. A. Katine, J. Langer, B. Ocker, I. N. Krivorotov, and K. L. Wang, Applied Physics Letters 104, 112410 (2014).

[36] R. Tomasello, K. Y. Guslienko, M. Ricci, A. Giordano, J. Barker, M. Carpentieri, O. Chubykalo-Fesenko, and G. Finocchio, Physical Review B 97, 060402(R) (2018).

[37] S. Schlotter, P. Agrawal, and G. S. D. Beach, Applied Physics Letters 113, 092402 (2018).

[38] A. A. Timopheev, R. Sousa, M. Chshiev, T. Nguyen, and 
B. Dieny, Scientific Reports 6, 26877 (2016).

[39] A. Slavin and V. Tiberkevich, IEEE Transactions on Magnetics 45, 1875 (2009).
[40] J. Barnaś, M. Gmitra, M. Misiorny, and V. Dugaev, physica status solidi (b) 244, 2304 (2007). 